\title{
Correction to: Computational Science and Its Applications - ICCSA 2021
}

\author{
Osvaldo Gervasi (D), Beniamino Murgante (D), Sanjay Misra (D), \\ Chiara Garau (D), Ivan Blečić (D), David Taniar (D), \\ Bernady O. Apduhan, Ana Maria A. C. Rocha (iD, \\ Eufemia Tarantino (D), and Carmelo Maria Torre (D)
}

\section{Correction to: \\ O. Gervasi et al. (Eds.): Computational Science and Its Applications - ICCSA 2021, LNCS 12954, https://doi.org/10.1007/978-3-030-86979-3}

In the originally published version, in the article "The Impact of COVID-19 Pandemic on the Perception of Public Transportation Users in Amman (Jordan)" (DOI: https:// doi.org/10.1007/978-3-030-86979-3_28) in Table 5, which is at Page 396 of the published book, the statement "(before the pandemic)" was added in the headings by mistake. The statement has been removed. The correct headings are "Number of walking days", "Number of days using the private car", "Number of days using public transportation", "Reasons for choosing the means of traveling".

In the originally published version of chapter 31, the name of one of the Authors was incorrect. The Author's name has been corrected as "Cézar Henrique Barra Rocha".

The updated version of these chapters can be found at https://doi.org/10.1007/978-3-030-86979-3_28

https://doi.org/10.1007/978-3-030-86979-3_31 